\title{
Magnetic moment of the $\rho$ meson in instant-form relativistic quantum mechanics
}

\author{
A. F. Krutov, ${ }^{1, *}$ R. G. Polezhaev, ${ }^{1, \dagger}$ and V. E. Troitsky ${ }^{2, *}$ \\ ${ }^{1}$ Samara University, 443086 Samara, Russia \\ ${ }^{2}$ D.V. Skobeltsyn Institute of Nuclear Physics, M. V. Lomonosov Moscow State University, \\ 119991 Moscow, Russia
}

(Received 5 January 2018; published 28 February 2018)

\begin{abstract}
We derive an explicit analytical expression for the magnetic dipole moment of the $\rho$ meson, $\mu_{\rho}$, in a relativistic constituent-quark model. We adopt our relativistic approach to composite systems, modified instant-form (mIF) relativistic quantum mechanics (RQM), that we used particularly to construct a unified $\pi \& \rho$ model [Phys. Rev. D 93, 036007 (2016)] describing electroweak properties of light mesons. This model provides a parameter-free calculation to give $\mu_{\rho}=2.16 \pm 0.03\left[e / 2 M_{\rho}\right]$ which is in accordance with the conventional experimental data. The magnetic, quadrupole, and charge form factors also are derived and presented. We consider the small uncertainty of our value of the magnetic moment as one of the undoubted advantages of the method. A comparison is made with recent lattice QCD results and previous calculations using a variety of methods.
\end{abstract}

DOI: 10.1103/PhysRevD.97.033007

\section{INTRODUCTION}

For our understanding of the structure of strong interaction, the electromagnetic form factors are of fundamental importance and give complementary information. Hadron form factors provide an important tool for understanding the structure of bound states in quantum chromodynamics (QCD). The study of electromagnetic properties of the $\pi$ and $\rho$ mesons consisting of two light $(u, d)$ quarks is of particular interest because in numerous respects they are the simplest bound states.

It is worth noting that the experimental situation is quite different in the cases of $\pi$ and $\rho$ mesons. The pion properties are well known from experiments. On the contrary, the experimental data on the $\rho$ meson are scarce. Its lifetime is very short, $\sim 4.5 \times 10^{-24} \mathrm{~s}$, so direct measurements of its electroweak properties (e.g., electromagnetic form factors and static moments) are nearly impossible.

Nevertheless, these form factors are important for hadron physics: for example, they contribute to meson exchange currents. They are also closely related to axial-vector diquark models of nucleon form factors. Besides, the description of

\footnotetext{
*krutov@ssau.ru polezaev@list.ru

troitsky@ theory.sinp.msu.ru
}

Published by the American Physical Society under the terms of the Creative Commons Attribution 4.0 International license. Further distribution of this work must maintain attribution to the author(s) and the published article's title, journal citation, and DOI. Funded by SCOAP ${ }^{3}$. the $\rho$ meson is of interest on its own account. Under such conditions, the role of theoretical investigations does increase. Indeed, recently the interest in $\rho$ meson form factors has been renewed. During recent years, a number of theoretical approaches to the structure of the $\rho$ meson appeared although their results cannot be directly compared to measurements. Below, we review these approaches while discussing the results of the calculations presented in Table I.

Theoretical approaches to the description of bound states are split into two directions. From the high-energy side, $\mathrm{QCD}$, which is widely believed to be a fundamental theory of the strong forces, becomes strongly coupled at the relevant energy scales, so trustworthy perturbative calculations help a little in the quantitative description of precise low-energy data, which there is no lack of. From the lowenergy side, a number of successful models to describe the data have been developed. To be quantitative, they necessarily require some phenomenological input. None of these models can be consistently and quantitatively derived from the QCD Lagrangian.

The approach that we use in the present paper is a particular relativistic formulation of constituent-quark model (CQM) that is based on the classical paper by P. Dirac [29] [so-called relativistic Hamiltonian dynamics (RHD) or relativistic quantum mechanics (RQM)]. RQM can be formulated in different ways or in different forms of dynamics. The main forms are instant form (IF), point form (PF) and light-front (LF) dynamics. The description of different forms of RQM dynamics can be found in the reviews [30-33]. Today, the approach is largely used for nonperturbative description of particle structure. It gives an opportunity to construct a 
TABLE I. The comparison of the results for the magnetic moment $\mu_{\rho}$ (in natural magnetons $e / 2 M_{\rho}$ ) in different approaches.

\begin{tabular}{lc}
\hline \hline Model & $\mu_{\rho}$ \\
\hline This work, mIF RHD & $2.16 \pm 0.03$ \\
Cardarelly, LF RHD [1] & 2.26 \\
Melo, LF RHD [2] & 2.14 \\
Bakker, LF RHD [3] & 2.1 \\
Jaus, LF RHD [4] & 1.83 \\
Choi, LF RHD [5] & 1.92 \\
He, LF, IF RHD [6] & 1.5 \\
He, PF RHD [6] & 0.9 \\
Biernat, PF RHD [7] & 2.20 \\
Sun, LF CQM [8] & 2.06 \\
Hawes, Dyson-Schwinger equation (DSE) [9] & 2.69 \\
Ivanov, DSE [10] & 2.44 \\
Bhagwat, DSE [11] & 2.01 \\
Roberts, DSE [12] & 2.11 \\
Pitschmann, DSE [13] & 2.11 \\
Carrillo-Serrano, Nambu-Jona-Lasinio & 2.59 \\
$\quad$ model (NJL) [14] & \\
Luan, NJL [15] & 2.1 \\
Samsonov, QCD sum rules [16] & $2.0 \pm 0.3$ \\
Aliev, QCD sum rules [17] & $2.4 \pm 0.4$ \\
Melikhov, LF triangle [18] & 2.35 \\
Šimonis, bag model [19] & 2.06 \\
Bagdasaryan, relativistic CQM [20] & 2.3 \\
Badalian, relativistic Hamiltonian [21] & 1.96 \\
Djukanovic, effective field theory [22] & 2.24 \\
Andersen, lattice [23] & $2.25 \pm 0.34$ \\
Hedditch, lattice [24] & $2.39 \pm 0.01$ \\
Lee, lattice [25] & $2.11 \pm 0.08$ \\
Owen, lattice [26] & 2.10 \\
Lushevskaya, lattice [27] & \\
Gudinõ, experiment [28] & \\
\hline \hline & \\
\hline
\end{tabular}

nonperturbative QCD model on the basis of constituentquark models. It is just such a model that we have constructed [34] - the unified $\pi \& \rho$ model with no free parameters remaining.

Now our goal is to obtain the value of the $\rho$ meson magnetic moment $\mu_{\rho}$ using explicit analytic formula which we derive here within the unified $\pi \& \rho$ model. It is extremely important to emphasize that our $\mu_{\rho}$ is obtained without any fitting parameters: in the unified $\pi \& \rho$ model [34] there remains no possibility to fit, all parameters are already fixed. Our result for the magnetic moment, $\mu_{\rho}=2.16 \pm 0.03$ $\left[e / 2 M_{\rho}\right]$, compares well with the conventional experimental result, but with significantly smaller uncertainty.

The model constructed in [34] is based on our version of RQM, the modified instant form (mIF) of RQM [35,36], and on the actual calculation of the $\pi$-meson structure [37] in 1998. This model has predicted with surprising accuracy the values of $F_{\pi}\left(Q^{2}\right)$, which were measured later in JLab experiments [38-42] (see discussion in Ref. [43]). Another advantage of the approach is matching with the QCD predictions in the ultraviolet limit: when consistent-quark masses are switched off, as expected at high energies, the model reproduces correctly not only the functional form of the QCD asymptotics, but also the numerical coefficient; see Refs. [44-46] for details. Let us note that mIF was successfully used also for other composite systems, namely, the deuteron [47-49] and the $K$-meson [50].

The rest of paper is organized as follows. In Sec. II we recall briefly the main points of our model. The $\rho$ meson electromagnetic current is written in terms of the Sachs form factors as well as in terms of form factors appropriate to the general method of relativistic invariant parametrization of matrix elements of local operators that we use. The brief description of the modified impulse approximation (MIA) is given. In Sec. III the $\rho$-meson magnetic moment is obtained. It is shown that the electromagmetic static moments of the $\rho$ meson are to be considered as regular generalized functions (distributions) determined on the space of test functions-quark-quark wave functions. To calculate the static moments one has to take the weak limits at $Q^{2} \rightarrow 0$ of corresponding functionals. The explicit analytic expression for the $\rho$ meson magnetic moment is obtained. We discuss the values of the parameters of the model which were defined previously in [34], and that we use here. The value of the calculated $\rho$ meson magnetic moment is given. This Section contains a discussion and the comparison of obtained results with the results of other authors. We briefly conclude in Sec. IV and present some details of the calculation in the Appendix.

\section{ELECTROMAGNETIC FORM FACTORS OF THE $\rho$ MESON IN MODIFIED INSTANT FORM RQM}

In this paper we use the relativistic constituent model that describes the hadron properties at the quark level in terms of degrees of freedom of constituent quarks. The constituent quarks are considered as extended objects, the internal characteristics of which (mean square radius, anomalous magnetic moments, form factors) are parameters of the model. As a relativistic variant of the constituent model we choose the method of RQM.

The RQM method, as a relativistic theory of composite systems, is based on the direct realization of the Poincare algebra on the set of dynamical observables on the Hilbert space. The RQM theory of particles lies between local field theoretic models and nonrelativistic quantum mechanical models.

Contrary to the field theory, RQM deals with a finite number of degrees of freedom from the very beginning. This is certainly a kind of a model approach. The preservation of the Poincaré algebra ensures the relativistic invariance. So, the covariance of the description in the frame of RQM is due to the existence of the unique unitary representation of the inhomogeneous group $S L(2, C)$ on the Hilbert space of composite system states with a finite number of degrees of freedom. 
The mathematics of RQM is similar to that of nonrelativistic quantum mechanics and permits one to assimilate the sophisticated methods of phenomenological potentials. It can be generalized to describe three or more particles. RQM is based on the simultaneous action of two fundamental principles, relativistic invariance and the Hamiltonian principle, and presents the most adequate tool to treat the systems with a finite number of degrees of freedom. The use of RQM enables one to separate the main degrees of freedom and thus to construct a convenient relativistic invariant approach to the electroweak structure of two-particle composite systems.

We use one of the forms of RQM, namely a version of the IF. The dynamics of a composite system, that is the interaction of the constituents is described in IF in the frame of the general RQM axiomatics. This means, particularly, that the inclusion of the interaction in the algebra of the Poincaré group is realized by means of the BakamjianThomas procedure [51] which permits to preserve the commutation relations between the generators of the group (the observables (see the reviews [31,33]). Following this procedure one includes the constituent-interaction operator by adding it to the operator of the mass of the free constituent system:

$$
\hat{M}_{0} \rightarrow \hat{M}_{I}=\hat{M}_{0}+\hat{V}
$$

Here $\hat{M}_{0}$ is the operator of the invariant mass of the system without interaction and $\hat{M}_{I}$ is the operator of the mass of interacting system. In IF dynamics, the interaction operator obeys the following conditions:

$$
\begin{gathered}
\hat{M}_{I}=\hat{M}_{I}^{+}, \quad \hat{M}_{I}>0, \\
{[\hat{\vec{P}}, \hat{V}]=[\hat{\vec{J}}, \hat{V}]=\left[\vec{\nabla}_{P}, \hat{V}\right]=0 .}
\end{gathered}
$$

The conditions (2) present spectral conditions for the mass operator. Equations (3) ensure that the algebraic relations of the Poincare algebra are fulfilled. The constraints (3) are not too restrictive. For example, they are satisfied by all nonrelativistic interaction potentials. Equations (3) mean, in particular, that the interaction potential does not depend on the total moment of the system. In RQM the wave function of the system of interacting particles is defined as the eigenfunction of a complete set of commuting operators. In IF this set is

$$
\hat{M}_{I}^{2} \quad\left(\text { or } \quad \hat{M}_{I}\right), \quad \hat{J}^{2}, \quad \hat{J}_{3}, \quad \hat{\vec{P}} \text {. }
$$

$\hat{J}^{2}$ is the operator of the square of the total angular momentum. In IF the operators $\hat{J}^{2}, \hat{J}_{3}, \hat{\vec{P}}$ coincide with those for the free system. So, in (4), only the operator $\hat{M}_{I}^{2}\left(\hat{M}_{I}\right)$ depends on the interaction.
To diagonalize the operators $\hat{J}^{2}, \hat{J}_{3}, \hat{\vec{P}}$, one has first to construct the adequate basis in the state space of composite system. In the case of two-particle system the Hilbert space in RQM is the direct product of two one-particle Hilbert spaces: $\mathcal{H}_{q \bar{q}} \equiv \mathcal{H}_{q} \otimes \mathcal{H}_{\bar{q}}$. As a basis in $\mathcal{H}_{q \bar{q}}$ one can choose the following set of two-particle state vectors where the motion of the center of mass is separated:

$$
\left|\vec{P}, \sqrt{s}, J, l, S, m_{J}\right\rangle .
$$

Here $P_{\mu}=\left(p_{1}+p_{2}\right)_{\mu}, P_{\mu}^{2}=s, \sqrt{s}$ is the invariant mass of the two-particle system, $l$ is the orbital angular momentum in the center-of-mass frame (C.M.S.), $\vec{S}^{2}=\left(\vec{S}_{1}+\vec{S}_{2}\right)^{2}=$ $S(S+1), S$ is the total spin in C.M.S., $J$ is the total angular momentum with the projection $m_{J}$, and $p_{1}, p_{2}$ are the constituent moments. The two-particle basis with separated motion of the center of mass (5) is connected with the basis of individual spins and momenta of two particles through the appropriate Clebsh-Gordan decomposition for the Poincaré group (see, e.g., [33]).

So, to obtain the system wave function in the basis (5) one needs to diagonalize the operator $\hat{M}_{I}^{2}$ (or $\hat{M}_{I}$ ). The eigenvalue problem for the operator $\hat{M}_{I}^{2}$ can be written in the form of nonrelativistic Schrödinger equation, the corresponding interaction operator having the meaning of a phenomenological nonrelativistic potential. The twoparticle wave function of relative motion for fixed total angular momentum is:

$\varphi_{l S}^{J}(k(s))=\sqrt[4]{s} u_{l S}(k) k, \quad \sum_{l S} \int u_{l S}^{2}(k) k^{2} d k=1$,

where $u_{l S}(k)$ is a model wave function which is a solution of the eigenvalue problem for the operator $\hat{M}_{I}^{2}$ or $\hat{M}_{I}$ in the representation given by the basis (5), $k=\sqrt{s-4 M^{2}} / 2$. The normalization factors that stay in (6) with $u_{l S}(k)$ correspond to the transition to the relativistic density of states:

$$
k^{2} d k \rightarrow \frac{k^{2} d k}{2 \sqrt{k^{2}+M^{2}}} .
$$

It is worth to notice that wave functions in RQM defined as the eigenfunctions of the operators set (4) in general are not the same as relativistic covariant wave functions defined as solutions of wave equations or as the matrix elements of a local Heisenberg field.

Our approach has a number of features that distinguish it from other forms of dynamics and other approaches in the frames of IF $[33,35,36]$. In particular, this approach (see, for example, $[33,36]$ ) differs essentially from that of traditional RQM in what concerns the method of construction of transition current operators. The main point of our approach to the construction of the electroweak current operator is the so-called method of the canonical 
parametrization of the local operator matrix elements. The foundation of the method was given in [52] and it was generalized to the case of composite systems in [53-55]. This parametrization is a realization of the Wigner-Eckart theorem for the Poincaré group [55] and so it enables one, for given matrix element of arbitrary tensor dimension, to separate the reduced matrix elements (form factors) that are invariant under the action of the Poincaré group. Matrix element of an operator is presented by a sum of terms which are the products of a covariant and an invariant parts. The covariant part of the matrix element describes its transformation (geometrical) properties while all the dynamical information about the transition is contained in the invariant part-in the reduced matrix elements, or form factors. In our approach, the reduced matrix elements are generalized functions. Strictly speaking, they are generalized functions in all cases of composite models, in particular, if one uses impulse approximation.

Our approach has the following characteristic features:

(a) The electroweak current matrix element satisfies automatically the relativistic covariance conditions and in the case of the electromagnetic current also the conservation law.

(b) We propose a modified impulse approximation (MIA), which is formulated in terms of reduced matrix elements and not in terms of operators as it takes place in standard impulse approximation. It is constructed in a relativistic invariant way. This means that our MIA does not depend on the choice of the coordinate frame, and this contrasts principally with the "frame-dependent" impulse approximation usually used in the instant form (IF) of dynamics.

(c) For composite systems (including the spin-1 case) the approach guarantees the uniqueness of the solution for form factors and does not use such concepts as "good" and "bad" current components.

The analytic properties of the pion form factor in the complex plane of the transfer momentum square in our model correspond to properties that follow from general principles of quantum field theory [56]. The model was also applied to the calculation of electroweak parameters of the $\rho$ meson [34], for which particularly interesting relations have been obtained. It is just those results which give the parameter-free method that permits to obtain the magnetic moment of the $\rho$ meson without any fitting.

The static electromagnetic moments of a particle are the limit values of Sachs form factors at $Q^{2} \rightarrow 0$. For spin-1 particles, including $\rho$ meson, the electromagnetic current matrix element can be written in terms of Sachs form factors in the Breit frame as follows (see, e.g., [57,58]):

$$
\begin{aligned}
\left\langle\vec{p}_{\rho}, m_{J}\left|j_{\mu}(0)\right| \vec{p}_{\rho}^{\prime}, m_{J}^{\prime}\right\rangle & =G^{\mu}\left(Q^{2}\right), \\
G^{0}\left(Q^{2}\right) & =2 p_{\rho 0}\left\{\left(\vec{\xi}^{\prime} \vec{\xi}^{*}\right) G_{C}\left(Q^{2}\right)+\left[\left(\vec{\xi}^{*} \vec{Q}\right)\left(\vec{\xi}^{\prime} \vec{Q}\right)-\frac{1}{3} Q^{2}\left(\vec{\xi}^{\prime} \vec{\xi}^{*}\right)\right] \frac{G_{Q}\left(Q^{2}\right)}{2 M_{\rho}^{2}}\right\}, \\
\vec{G}\left(Q^{2}\right) & =\frac{p_{\rho 0}}{M_{\rho}}\left[\vec{\xi}^{\prime}\left(\vec{\xi}^{*} \vec{Q}\right)-\vec{\xi}^{*}\left(\vec{\xi}^{\prime} \vec{Q}\right)\right] G_{M}\left(Q^{2}\right) .
\end{aligned}
$$

Here $G_{C}, G_{Q}, G_{M}$ are the charge, quadrupole and magnetic form factors, respectively,

$$
\begin{aligned}
q^{\mu} & =\left(p_{\rho}-p_{\rho}^{\prime}\right)^{\mu}=(0, \vec{Q}), \\
p_{\rho}^{\mu} & =\left(p_{\rho 0}, \frac{1}{2} \vec{Q}\right), \quad p_{\rho}^{\prime \mu}=\left(p_{\rho 0},-\frac{1}{2} \vec{Q}\right), \\
p_{\rho 0} & =\sqrt{M_{\rho}^{2}+\frac{1}{4} Q^{2}}, \quad \vec{Q}=(0,0, Q) . \\
\xi^{\mu}( \pm 1) & =\frac{1}{\sqrt{2}}(0, \mp 1,-i, 0), \\
\xi^{\mu}(0) & =(0,0,0,1) .
\end{aligned}
$$

The arguments of the polarization vector $\xi$ are the projections of the total angular momentum. The static limits of form factors in (8) give the magnetic $\mu_{\rho}$ (in units $e / 2 M_{\rho}$ ) and the quadrupole $Q_{\rho}$ moments [58]:

$$
G_{M}(0)=\mu_{\rho}, \quad G_{Q}(0)=M_{\rho}^{2} Q_{\rho} .
$$

One can use the general procedure of relativistic covariant construction of local operators matrix elements to obtain, in our version of IF RQM, the current matrix element (8) in an arbitrary coordinate system [36]:

$$
\begin{aligned}
\left\langle\vec{p}_{c}, m_{J \rho}\left|j_{\mu}(0)\right| \vec{p}_{\rho}^{\prime}, m_{J \rho}^{\prime}\right\rangle & =\left\langle m_{J \rho}\left|D^{1}\left(p_{\rho}, p_{\rho}^{\prime}\right) \sum_{i=1,3} \tilde{\mathcal{F}}_{p}^{i}(t) \tilde{A}_{\mu}^{i}\right| m_{J \rho}^{\prime}\right\rangle, \\
\tilde{\mathcal{F}}_{c}^{1}(t)= & \tilde{f}_{10}^{\rho}+\tilde{f}_{12}^{\rho}\left\{\left[i p_{\rho_{\nu}} \Gamma^{\nu}\left(p_{\rho}^{\prime}\right)\right]^{2}-\frac{1}{3} \operatorname{Sp}\left[i p_{\rho_{\nu}} \Gamma^{\nu}\left(p_{\rho}^{\prime}\right)\right]^{2}\right\} \\
& \times \frac{2}{\operatorname{Sp}\left[p_{\rho_{\nu}} \Gamma^{\nu}\left(p_{\rho}^{\prime}\right)\right]^{2}} \\
\tilde{\mathcal{F}}_{\rho}^{3}(t)= & \tilde{f}_{30}^{\rho}, \\
\tilde{A}_{\mu}^{1}= & \left(p_{\rho}+p_{\rho}^{\prime}\right)_{\mu}, \\
\tilde{A}_{\mu}^{3}= & \frac{i}{M_{\rho}} \varepsilon_{\mu \nu \lambda \sigma} p_{\rho}^{\nu} p_{\rho}^{\prime \lambda} \Gamma^{\sigma}\left(p_{\rho}^{\prime}\right) .
\end{aligned}
$$


Here $p_{\rho}^{\prime}, p_{\rho}$ are 4-momenta of the $\rho$ meson in initial and final states, respectively, $m_{J \rho}^{\prime}, m_{J \rho}$ are projections of the total angular momenta, $D^{1}\left(p_{\rho}, p_{\rho}^{\prime}\right)$ is the matrix of Wigner rotation, $M_{\rho}$ is the $\rho$ meson mass, $\tilde{f}_{10}^{\rho}, \tilde{f}_{12}^{\rho}, \tilde{f}_{30}^{\rho}$ are the charge, quadrupole and magnetic form factors of the $\rho$ meson, respectively.

The spin 4 -vector $\Gamma^{\nu}\left(p_{\rho}\right)$ is (see, e.g., [33]):

$$
\begin{aligned}
\Gamma_{0}\left(p_{\rho}\right) & =\left(\vec{p}_{\rho} \vec{j}\right), \\
\vec{\Gamma}\left(p_{\rho}\right) & =M_{\rho} \vec{j}+\frac{\vec{p}_{\rho}\left(\vec{p}_{\rho} \vec{j}\right)}{p_{\rho_{0}}+M_{\rho}}, \\
\Gamma^{2} & =-M_{\rho}^{2} j(j+1) .
\end{aligned}
$$

One can easily obtain the relation between the form factors in the form (10) and Sachs form factors (8):

$$
\begin{aligned}
G_{C}\left(Q^{2}\right) & =\tilde{f}_{10}^{\rho}\left(Q^{2}\right), \\
G_{Q}\left(Q^{2}\right) & =\frac{2 M_{\rho}^{2}}{Q^{2}} \tilde{f}_{12}^{\rho}\left(Q^{2}\right), \\
G_{M}\left(Q^{2}\right) & =-M_{\rho} \tilde{f}_{30}^{\rho}\left(Q^{2}\right) .
\end{aligned}
$$

In the paper [36] the form factors (10) were presented in the form of double integrals:

$$
\tilde{f}_{\text {in }}^{\rho}\left(Q^{2}\right)=\int d \sqrt{s} d \sqrt{s^{\prime}} \varphi(s) \tilde{G}_{\mathrm{in}}\left(s, Q^{2}, s^{\prime}\right) \varphi\left(s^{\prime}\right),
$$

where $\varphi(s)$ is the quarks wave function in the $\rho$ meson in the sense of RQM, $\tilde{G}_{\text {in }}\left(s, Q^{2}, s^{\prime}\right)$ are the reduced matrix elements on the Poincaré group. They are Lorentz-invariant regular generalized functions.

It is worth to note that while obtaining (13) no assumption or approximation concerning the form of the electromagnetic current operator was made. We have not assumed, in particular, that the current operator is a sum of the one-particle current operators of the constituents, that means that we have not used the so-called impulse approximation which is known to break the Lorentzcovariance and the conservation law for the compositesystem electromagnetic current in IF RQM. So, for (13) the Lorentz-covariance and current conservation law are valid.

In general, the explicit form of the functions $\tilde{G}_{\text {in }}\left(s, Q^{2}, s^{\prime}\right)$, is unknown. To calculate these functions we propose a modified impulse approximation (MIA). In contrast to the generally accepted impulse approximation MIA is formulated in terms of reduced matrix elements on the Poincaré group (form factors) extracted from the current matrix element and not in terms of current operators themselves. For (13), MIA means that the reduced matrix elements $\tilde{G}_{\text {in }}\left(s, Q^{2}, s^{\prime}\right)$ are changed for the free two-particle form factors with no interaction between components. Such free form factors enter the electromagnetic current matrix element for a system of two free particles with $\rho$-meson quantum numbers. The corresponding formulas for these form factors are given in the Appendix. Note that the current matrix element as a whole still contains some contributions of twoparticle currents in a way which such that ensures its correct transformation properties.

Using the relations (12) we derive the integral representations for the $\rho$-meson Sachs form factors in MIA:

$$
\begin{aligned}
G_{C}\left(Q^{2}\right) & =\int d \sqrt{s} d \sqrt{s^{\prime}} \varphi(s) g_{0 C}\left(s, Q^{2}, s^{\prime}\right) \varphi\left(s^{\prime}\right), \\
G_{Q}\left(Q^{2}\right) & =\frac{2 M_{\rho}^{2}}{Q^{2}} \int d \sqrt{s} d \sqrt{s^{\prime}} \varphi(s) g_{0 Q}\left(s, Q^{2}, s^{\prime}\right) \varphi\left(s^{\prime}\right), \\
G_{M}\left(Q^{2}\right) & =-M_{\rho} \int d \sqrt{s} d \sqrt{s^{\prime}} \varphi(s) g_{0 M}\left(s, Q^{2}, s^{\prime}\right) \varphi\left(s^{\prime}\right),
\end{aligned}
$$

where $g_{0 C}, g_{0 Q}, g_{0 M}$ are the free two-particle charge, quadrupole and magnetic form factors for the system of two free fermions with quantum numbers of $\rho$ meson respectively (the explicit expression for these form factors are given in Appendix), $\varphi(s)$ is the two-quark $\rho$-meson wave function in the sense of RQM,

$$
\varphi(s)=\sqrt[4]{s} \psi(k) k, \quad k=\frac{1}{2} \sqrt{s-4 M^{2}}
$$

with the normalization condition:

$$
\int k^{2} \psi^{2}(k) d k=1
$$

As was shown in [35] the free two-particle electromagnetic form factor is a regular generalized function (distribution) defined on the space of test functions in $\mathbf{R}^{2}, Q^{2}$ is the parameter of the generalized function. This generalized function giving the $\rho$ meson form factor is given by the functional $\left\langle g_{0 i}\left(s, Q^{2}, s^{\prime}\right), \phi\left(s, s^{\prime}\right)\right\rangle, i=C, Q, M$ that is defined as an integral of the product of the function $g_{0 i}\left(s, Q^{2}, s^{\prime}\right)$ and the test function $\phi\left(s, s^{\prime}\right)$. If we take the product of quark wave functions in the initial and the final states for the test function, then the considered functional is the corresponding $\rho$-meson form factor.

\section{THE $\rho$-MESON MAGNETIC MOMENT IN $\pi \& \rho$ MODEL. RESULTS AND DISCUSSION}

To calculate the $\rho$-meson magnetic moment in our approach we have to take the static limit of the form factor (14) and to choose the values of the parameters entering our equations. The limit is to be taken in the weak sense and there is no need in fitting the parameters: all of them were fixed in our $\pi \& \rho$ model in our previous work [34].

It is easy to see that the function (14) has no strong limit at $Q^{2} \rightarrow 0$. As the function is a generalized function one 
has to take the limit in the weak sense. So, the (weak) static limit of (14) gives the following $\rho$-meson magnetic moment:

$$
\begin{aligned}
\mu_{\rho}= & \frac{M_{\rho}}{2 M} \int_{2 M}^{\infty} d \sqrt{s} \frac{\varphi^{2}(s)}{\sqrt{s-4 M^{2}}} \\
& \times\left\{1-L(s)+\left(\kappa_{u}+\kappa_{\bar{d}}^{-}\right)\left[1-\frac{1}{2} L(s)\right]\right\}, \\
L(s)= & \frac{2 M^{2}}{\sqrt{s-4 M^{2}}(\sqrt{s}+2 M)} \\
& \times\left[\frac{1}{2 M^{2}} \sqrt{s\left(s-4 M^{2}\right)}+\ln \frac{\sqrt{s}-\sqrt{s-4 M^{2}}}{\sqrt{s}+\sqrt{s-4 M^{2}}}\right],
\end{aligned}
$$

where $\kappa_{u}, \kappa_{\bar{d}}$ are anomalous magnetic moments of $u$ - and $\bar{d}$-quarks.

It is of interest to separate the contribution to (16) of purely relativistic kinematic effect of the Wigner rotation of quark spins $[35,36]$ that appears under relativistic invariant spin summation. If we put $\omega_{1}=\omega_{2}=0$ in the free twoparticle magnetic form factor (see Appendix) we obtain the magnetic moment without taking into account the Wigner spin rotation in the form:

$$
\begin{aligned}
\tilde{\mu}_{\rho}= & \frac{M_{\rho}}{2 M}\left(1+\kappa_{u}+\kappa_{\bar{d}}\right) \\
& \times \int_{2 M}^{\infty} d \sqrt{s} \frac{\varphi^{2}(s)}{\sqrt{s-4 M^{2}}}\left\{1-\frac{1}{2} L(s)\right\},
\end{aligned}
$$

The spin-rotation contribution is negative and its value is $\approx 15 \%$ of that of the $\rho$-meson magnetic moment.

To calculate the numerical value of the $\rho$-meson magnetic moment (16) we use for the wave functions in the sense of RQM (15) the following model wave function that depends on only one parameter and is largely used in relativistic composite quark model (on a level with harmonic oscillator wave function) [59] (see, also [60]), the power-law wave functions:

$$
\psi(k)=N_{P L}\left(k^{2} / b_{\rho}^{2}+1\right)^{-n}, \quad n=2,3 .
$$

The parameters that enter our calculation in whole are from two groups:

(1) the parameters that describe the constituent quarks per se (the quark mass $M$, the anomalous magnetic moments of quarks $\kappa_{q}$, that enter our formulas through the sum $s_{q}=\kappa_{u}+\kappa_{\bar{d}}$, and the quark mean square radius (MSR) $\left\langle r_{q}^{2}\right\rangle$ );

(2) the parameter $b_{\rho}$ that enters the quark wave function (18) and is determined by the quark interaction potential.

As it was shown in [34], to calculate electroweak properties of the $\rho$ meson, one can use the same values of quark parameters from the first group as were used for the pion [37]. In the paper [37] on the pion, we have shown that in our approach all the parameters of the first group are the functions of the quark mass $M$ and are defined by its value. In particular, for the quark MSR we can use the relation (see, also [61])

$$
\left\langle r_{q}^{2}\right\rangle \simeq 0.3 / M^{2} .
$$

In our $\pi \& \rho$ model [34], the mass of the constituent $u-\bar{d}$ quarks was chosen to be $M=0.22 \mathrm{GeV}$. The sum of the anomalous magnetic moments of quarks was taken as $s_{q}=0.0268$ in quark magnetons. It is just these values of the parameters that in the frame of our version of IF RQM provide the successful and predictive description of pion properties [37,43], as it was mentioned in the Introduction.

For the parameter $b_{\rho}$ of the wave function, we use the value obtained in our paper [34] from the fitting of the lepton decay constant of the $\rho$ meson. Using the wave function (18) with $n=3$ we have obtained $b_{\rho}=$ $(0.385 \pm 0.019) \mathrm{GeV}$. The uncertainty is due to the uncertainty of the experimental value of the decay constant $f_{\rho}^{\exp }=(152 \pm 8) \mathrm{MeV}[62,63]$. The calculation of [34] schematically can be presented as a chain,

$$
f_{\rho}^{\exp } \rightarrow b_{\rho} \rightarrow\left\langle r_{\rho}^{2}\right\rangle,
$$

and the obtained value $\left\langle r_{\rho}^{2}\right\rangle=(0.56 \pm 0.04) \mathrm{fm}^{2}$, one can consider as a theoretical extraction of the experimental value (see, e.g., [64]).

The electromagnetic form factors of constituent quarks are taken in the form $[33,44,45,65]$

$$
\begin{aligned}
G_{E}^{q}\left(Q^{2}\right) & =e_{q} f_{q}\left(Q^{2}\right), \\
G_{M}^{q}\left(Q^{2}\right) & =\left(e_{q}+\kappa_{q}\right) f_{q}\left(Q^{2}\right),
\end{aligned}
$$

where $e_{q}$ is the quark charge and $\kappa_{q}$ is the quark anomalous magnetic moment. The quark form factor has the form [65]

$$
f_{q}\left(Q^{2}\right)=\frac{1}{1+\ln \left(1+\left\langle r_{q}^{2}\right\rangle Q^{2} / 6\right)},
$$

where $\left\langle r_{q}^{2}\right\rangle$ is the MSR of the constituent quark. Values of all parameters used in these expressions are taken from the $\pi$ meson calculation. As we have mentioned, these values give the pion form factor asymptotics at large momentum transfer that coincides with that of QCD (see e.g., Ref. [45]).

In Figs. 1-3, the results for the electromagnetic $\rho$-meson form factors obtained with the parameters described above are presented. For comparison, the results from some other papers are given. Our quadrupole form factor differs strongly from that of other given results. In this context it is necessary to note that we (as opposed to, e.g., [5]) take into account the quark structure in terms of quark form factors (21), (22), that accelerating the decay of form 


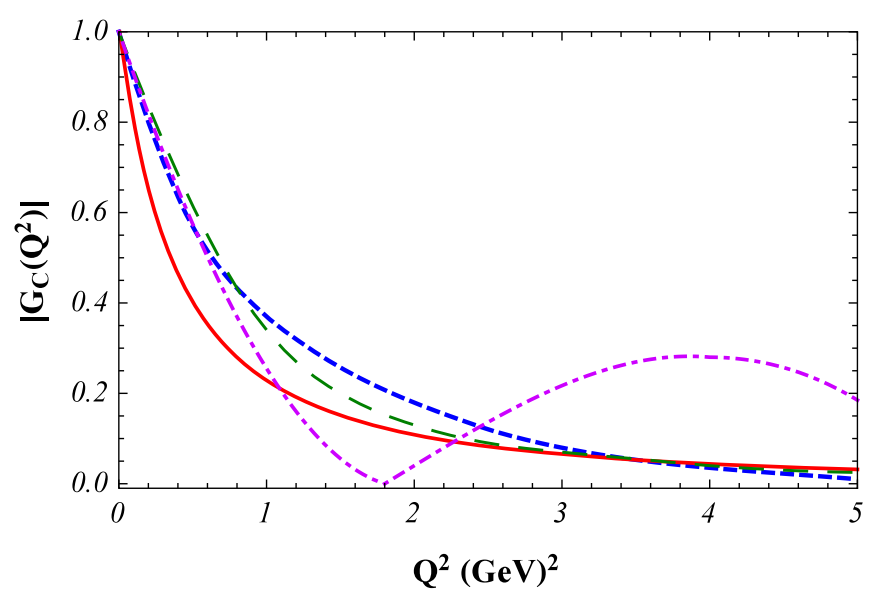

FIG. 1. Modulus of the charge $\rho$ meson form factor. Solid line (red): the results of our calculations with wave function (18) at $n=3$ and parameters described in the text, dashed line (green): the results of calculation in point-form dynamics [7], shortdashed line (blue): the result of calculations in light-front dynamics [5], dot-dashed line (violet): the results of calculation in the Dyson-Schwinger equation approach [9].

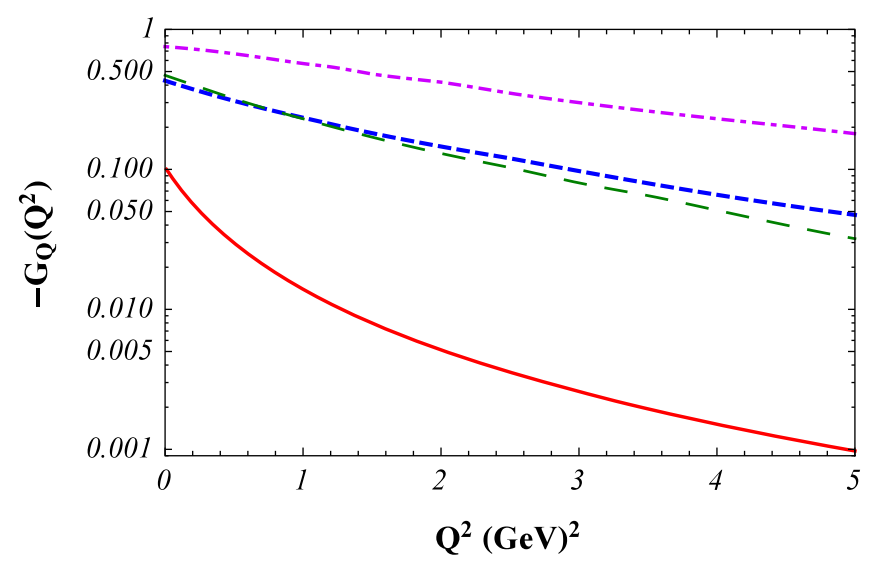

FIG. 2. The quadrupole $\rho$ meson form factor in the logarithmic scale, legend as in Fig. 1.

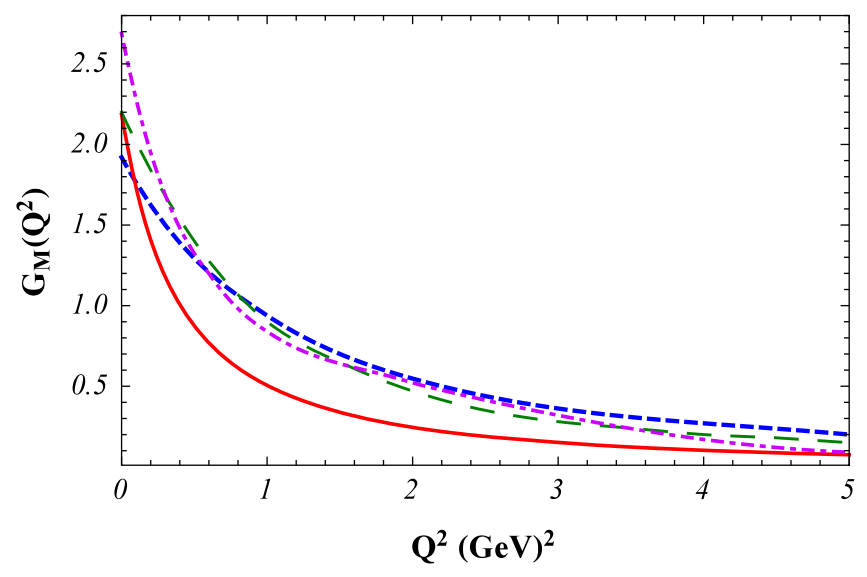

FIG. 3. The magnetic $\rho$ meson form factor, legend as in Fig. 1. factors. Note that the calculation using the formulas analogous to (14) described well the electron-deuteron polarization scattering [47] and so the experimental deuteron quadrupole form factor.

Finally, using the mentioned values of the parameters of the $\pi \& \rho$ model, we obtain from (16) the following value of the magnetic moment

$$
\mu_{\rho}=2.16 \pm 0.03\left[e / 2 M_{\rho}\right]
$$

which is in accordance with the conventional experimental data.

During the last decade, a number of papers appeared that considered the electroweak properties of $\rho$ meson according to the growing interest to the problem. A lot of theoretical information is obtained while the experimental base remains scare. We list below only the papers where the value of the $\rho$-meson magnetic moment is given whereas a lot of interesting papers on $\rho$ meson remain out of scope, for example those based on the holographic approach [66].

First, we refer to different formulation of RQM in the frame of relativistic composite model (see, e.g., [1-8,67]. These approaches are the most close in spirit to our approach. There are approaches based on the DysonSchwinger equation [9-13], on the Nambu-Jona-Lasinio model $[14,15]$. Some authors use QCD sum rules [16,17], Feynmann diagrams in the light front formalism [18], the bag model [19], relativistic composite model [20], the constructed relativistic Hamiltonian [21], a low-energy effective field theory [22], or lattice QCD calculations [23-27]. In the paper [28] the $\rho$ meson magnetic moment is obtained from $B A B A R$ cross section data for the reaction $e^{+} e^{-} \rightarrow \pi^{+} \pi^{-} 2 \pi^{0}$. They found the values using preliminary data from the $B A B A R$ Collaboration for the $\mu_{\rho}^{\text {exper }}=$ $2.1 \pm 0.5\left[e / 2 M_{\rho}\right]$. In a recent paper, $B A B A R$ Collaboration presents [68] the new experimental results concerning the mentioned reaction. One can hope that the new data processing would make it possible to increase the precision of the value of $\mu_{\rho}$ extracted from the experimental data.

The values of the $\rho$-meson magnetic moment in different approaches are presented in Table I.

One can see from Table I that the majority of results is grouped close to the value $\mu_{\rho}=2 e / 2 M_{\rho}$, that is near the gyromagnetic ratio $g=2$. The authors of the paper [69] noted, that universal closeness to $g=2$ may be also understood in comparison to the equivalence principle for vector mesons.

Let us discuss briefly our results. We emphasize once again that our calculation of the magnetic moment in the frame of the $\pi \& \rho$ model is parameter-free and is in very good accordance with experiment. The given value of the uncertainty of our result is totally due to the uncertainty of the experimental data for $f_{\rho}$. So, the refinement of this data will bring about the decrease of the uncertainty of our result for $\mu_{\rho}$. 
It is worth to notice that the $\pi \& \rho$ model gives the value of the charge $\rho$ - meson radius [34] in accordance with the Wu-Yang hypothesis [70] (see also Refs. [71-74]).

\section{CONCLUSIONS}

To summarize, we calculate the magnetic dipole moment of the $\rho$ meson, $\mu_{\rho}$, in relativistic constituent-quark model. We derive explicit analytical expression for $\mu_{\rho}$ exploiting our relativistic approach to composite systems, a version of the instant-form relativistic quantum mechanics. We have used this approach in the paper [34] to construct a unified $\pi \& \rho$ model describing electroweak properties of light mesons. In the present paper we calculate $\mu_{\rho}$ using our unified $\pi \& \rho$ model without addition of any fitting parameters. This parameter-free calculation gives the value contribution of the Wigner spin rotation to the magnetic moment $\mu_{\rho}$ is $(\approx 15 \%)$. We consider the small uncertainty $(\approx 1.4 \%)$. of our value of magnetic moment as one of undoubted advantages of the method. A comparison is made with recent lattice QCD results and other calculations using variety of methods.

So, we can state that in the frame of our $\pi \& \rho$ model, that is in our version of IF RQM approach and at the same common values of quark parameters, the concordant description of electroweak properties of the $\pi$ and $\rho$ mesons is obtained. The constructed relativistic approach demonstrated the predictability in describing the pion electromagnetic form factors (see our paper [43]) and gives, in accordance with experimental data, the electroweak characteristics of the pion as well as of the $\rho$ meson.

\section{ACKNOWLEDGMENTS}

V.E. T. is grateful to S. V. Troitsky for helpful discussions and valuable comments.

\section{APPENDIX: FREE TWO-PARTICLE FORM FACTORS}

The charge $g_{0 C}$, quadrupole $g_{0 Q}$, and magnetic $g_{0 M}$ form factors for free two-particle system are

$$
\begin{aligned}
g_{0 C}\left(s, Q^{2}, s^{\prime}\right)= & \frac{1}{3} R\left(s, Q^{2}, s^{\prime}\right) Q^{2} \\
& \times\left\{\left(s+s^{\prime}+Q^{2}\right)\left(G_{E}^{u}\left(Q^{2}\right)+G_{E}^{\bar{d}}\left(Q^{2}\right)\right)\right. \\
& \times\left[2 \cos \left(\omega_{1}-\omega_{2}\right)+\cos \left(\omega_{1}+\omega_{2}\right)\right] \\
& -\frac{1}{M} \xi\left(s, Q^{2}, s^{\prime}\right)\left(G_{M}^{u}\left(Q^{2}\right)+G_{M}^{\bar{d}}\left(Q^{2}\right)\right) \\
& \left.\times\left[2 \sin \left(\omega_{1}-\omega_{2}\right)-\sin \left(\omega_{1}+\omega_{2}\right)\right]\right\} ;
\end{aligned}
$$

$$
\begin{aligned}
g_{0 Q}\left(s, Q^{2}, s^{\prime}\right)= & \frac{1}{2} R\left(s, Q^{2}, s^{\prime}\right) Q^{2} \\
& \times\left\{\left(s+s^{\prime}+Q^{2}\right)\left(G_{E}^{u}\left(Q^{2}\right)+G_{E}^{\bar{d}}\left(Q^{2}\right)\right)\right. \\
& \times\left[\cos \left(\omega_{1}-\omega_{2}\right)-\cos \left(\omega_{1}+\omega_{2}\right)\right] \\
& -\frac{1}{M} \xi\left(s, Q^{2}, s^{\prime}\right)\left(G_{M}^{u}\left(Q^{2}\right)+G_{M}^{\bar{d}}\left(Q^{2}\right)\right) \\
& \left.\times\left[\sin \left(\omega_{1}-\omega_{2}\right)+\sin \left(\omega_{1}+\omega_{2}\right)\right]\right\} ;
\end{aligned}
$$

$$
\begin{aligned}
g_{0 M}\left(s, Q^{2}, s^{\prime}\right)= & -2 R\left(s, Q^{2}, s^{\prime}\right)\left\{\xi\left(s, Q^{2}, s^{\prime}\right)\left[G_{E}^{u}\left(Q^{2}\right)+G_{E}^{\bar{d}}\left(Q^{2}\right)\right] \sin \left(\omega_{1}-\omega_{2}\right)+\frac{1}{4 M}\left[G_{M}^{u}\left(Q^{2}\right)+G_{M}^{\bar{d}}\left(Q^{2}\right)\right]\right. \\
& \times\left[\left(s+s^{\prime}+Q^{2}\right) Q^{2}\left(\frac{3}{2} \cos \left(\omega_{1}-\omega_{2}\right)+\frac{1}{2} \cos \left(\omega_{1}+\omega_{2}\right)\right)-\frac{1}{4} \xi\left(s, Q^{2}, s^{\prime}\right)\right. \\
& \times\left[\frac{\left(\sqrt{s^{\prime}}+2 M\right)\left(s-s^{\prime}+Q^{2}\right)+\left(s^{\prime}-s+Q^{2}\right) \sqrt{s^{\prime}}}{\sqrt{s^{\prime}}\left(\sqrt{s^{\prime}}+2 M\right)}+\frac{(\sqrt{s}+2 M)\left(s^{\prime}-s+Q^{2}\right)+\left(s-s^{\prime}+Q^{2}\right) \sqrt{s}}{\sqrt{s}(\sqrt{s}+2 M)}\right] \\
& \times\left[\sin \left(\omega_{1}-\omega_{2}\right)-\sin \left(\omega_{1}+\omega_{2}\right)\right]-\frac{1}{2} \xi^{2}\left(s, Q^{2}, s^{\prime}\right)\left[\frac{1}{\sqrt{s^{\prime}}\left(\sqrt{s^{\prime}}+2 M\right)}+\frac{1}{\sqrt{s}(\sqrt{s}+2 M)}\right] \\
& \left.\left.\times\left[\cos \left(\omega_{1}-\omega_{2}\right)+\cos \left(\omega_{1}+\omega_{2}\right)\right]\right]\right\} .
\end{aligned}
$$

Here,

$$
\begin{aligned}
& R\left(s, Q^{2}, s^{\prime}\right)=\frac{\left(s+s^{\prime}+Q^{2}\right)}{2 \sqrt{\left(s-4 M^{2}\right)\left(s^{\prime}-4 M^{2}\right)}} \frac{\vartheta\left(s, Q^{2}, s^{\prime}\right)}{\left[\lambda\left(s,-Q^{2}, s^{\prime}\right)\right]^{3 / 2}} \frac{1}{\sqrt{1+Q^{2} / 4 M^{2}}}, \\
& \xi\left(s, Q^{2}, s^{\prime}\right)=\sqrt{s s^{\prime} Q^{2}-M^{2} \lambda\left(s,-Q^{2}, s^{\prime}\right)}
\end{aligned}
$$

and $\omega_{1}$ and $\omega_{2}$ are the Wigner rotation parameters, 


$$
\begin{aligned}
& \omega_{1}=\arctan \frac{\xi\left(s, Q^{2}, s^{\prime}\right)}{M\left[\left(\sqrt{s}+\sqrt{s^{\prime}}\right)^{2}+Q^{2}\right]+\sqrt{s s^{\prime}}\left(\sqrt{s}+\sqrt{s^{\prime}}\right)}, \\
& \omega_{2}=\arctan \frac{\alpha\left(s, s^{\prime}\right) \xi\left(s, Q^{2}, s^{\prime}\right)}{M\left(s+s^{\prime}+Q^{2}\right) \alpha\left(s, s^{\prime}\right)+\sqrt{s s^{\prime}}\left(4 M^{2}+Q^{2}\right)},
\end{aligned}
$$

$\alpha\left(s, s^{\prime}\right)=2 M+\sqrt{s}+\sqrt{s^{\prime}}, \vartheta\left(s, Q^{2}, s^{\prime}\right)=\theta\left(s^{\prime}-s_{1}\right)-\theta\left(s^{\prime}-s_{2}\right), \theta$ is the step-function,

$$
s_{1,2}=2 M^{2}+\frac{1}{2 M^{2}}\left(2 M^{2}+Q^{2}\right)\left(s-2 M^{2}\right) \mp \frac{1}{2 M^{2}} \sqrt{Q^{2}\left(Q^{2}+4 M^{2}\right) s\left(s-4 M^{2}\right)},
$$

and $M$ - the mass of $u$-and $\bar{d}$ quarks. The functions $s_{1,2}\left(s, Q^{2}\right)$ give the kinematically available region in the plane $\left(s, s^{\prime}\right)$. $G_{E, M}^{u, \bar{d}}\left(Q^{2}\right)$-is the Sachs form factors of $u$-and $\bar{d}$ quarks.

[1] F. Cardarelli, I. L. Grach, I. M. Narodetskii, G. Salmé, and S. Simula, Electromagnetic form factors of the $\rho$ meson in a light-front constituent quark model, Phys. Lett. B 349, 393 (1995).

[2] J. P. B. C. de Melo and T. Frederico, Covariant and lightfront approaches to the $\rho$ meson electromagnetic form factors, Phys. Rev. C 55, 2043 (1997).

[3] B. L. G. Bakker, H.-M. Choi, and C.-R. Ji, The vector meson form-factor analysis in light front dynamics, Phys. Rev. D 65, 116001 (2002).

[4] W. Jaus, Consistent treatment of spin-1 mesons in light-front quark model, Phys. Rev. D 67, 094010 (2003).

[5] H.-M. Choi and C.-R. Ji, Electromagnetic structure of the $\rho$ meson in the light-front quark model, Phys. Rev. D 70, 053015 (2004).

[6] J. He, B. Juliá-Díaz, and Y.-B. Dong, Electromagnetic form factors of pion and $\rho$ meson in the three forms of relativistic kinematics, Phys. Lett. B 602, 212 (2004).

[7] E. P. Biernat and W. Schweiger, Electromagnetic $\rho$ meson form factors in point-form relativistic quantum mechanics, Phys. Rev. C 89, 055205 (2014).

[8] B.-D. Sun and Y.-B. Dong, $\rho$ meson unpolarized generalized parton distrbutions with a light-front constituent quark model, Phys. Rev. D 96, 036019 (2017).

[9] E. T. Hawes and M. A. Pichowsky, Electromagnetic form factors of light vector mesons, Phys. Rev. C 59, 1743 (1999).

[10] M. A. Ivanov, Yu. I. Kalinovsky, and C. D. Roberts, Survey of heavy-meson observables, Phys. Rev. D 60, 034018 (1999).

[11] M. S. Bhagwat and P. Maris, Vector meson form factors and their quark-mass dependence, Phys. Rev. C 77, 025203 (2008).

[12] H. L. L. Roberts, A. Bashir, L. X. Gutierrez-Guerrero, C. D. Roberts, and D. J. Wilson, $\pi$ - and $\rho$ mesons, and their diquark partners, from a contact interaction, Phys. Rev. C 83, 065206 (2011).

[13] M. Pitschmann, C-Y. Seng, M. J. Ramsey-Musolf, C. D. Roberts, and D. J. Wilson, Electrc dipole moment of the $\rho$ meson, Phys. Rev. C 87, 015205 (2013).
[14] M. E. Carrillo-Serrano, W. Bentz, I. C. Cloët, and A. W. Thomas, Rho meson form factors in confining NambuJona-Lasino model, Phys. Rev. C 92, 015212 (2015).

[15] Y.-L. Luan, X.-L. Chen, and W.-Z. Deng, Meson ElectroMagnetic Form Factors in an Extended Nambu-JonaLasinio model including Heavy Quark Flavors, Chin. Phys. C 39, 113103 (2015).

[16] A. Samsonov, Magnetic moment of the $\rho$ meson in QCD sum rules: $\alpha_{s}$-corrections, J. High Energy Phys. 12 (2003) 061.

[17] T. M. Aliev, A. Özpineci, and M. Savci, Magnetic and quadrupole moments of light spin-1 mesons in light cone QCD sum rules, Phys. Lett. B 678, 470 (2009).

[18] D. Melikhov and S. Simula, Electromagnetic form factors in the light-front formalism and the Feynman triangle diagram: Spin-0 and spin-1 two-fermion systems, Phys. Rev. D 65, 094043 (2002).

[19] V. Śmonis, Magnetic properties of ground-state mesons, Eur. Phys. J. A 52, 90 (2016).

[20] A. S. Bagdasaryan, S. V. Esaibegian, and N. L. Ter-Isaakian, Form factors of meson and resonances at small and intermediate momentum transfers $Q^{2}$ in the relativistic quark models, Yad. Fiz. 42, 440 (1985) [Sov. J. Nucl. Phys. 42, 278 (1985)].

[21] A. M. Badalian and Yu. A. Simonov, Magnetic moments of mesons, Phys. Rev. D 87, 074012 (2013).

[22] D. Djukanovic, E. Epelbaum, J. Gegelia, and U.-G. Meißner, The magnetic moment of the $\rho$ meson, Phys. Lett. B 730, 115 (2014).

[23] W. Andersen and W. Wilcox, Lattice charge overlap. I. Elastic limit of $\pi$ and $\rho$ mesons, Ann. Phys. (N.Y.) 255, 34 (1997).

[24] J. N. Hedditch, W. Kamleh, B. G. Lasscock, D. B. Leinweber, A. G. Williams, and J. M. Zanotti, Pseudoscalar and vector meson form factors from lattice QCD, Phys. Rev. D 75, 094504 (2007).

[25] F. X. Lee, S. Moerschbachor, and W. Wilcox, Magnetic moments of vector, axial, and tensor mesons in lattice QCD, Phys. Rev. D 78, 094502 (2008). 
[26] B. Owen, W. Kamel, D. Leinweber, B. Menadue, and S. Mahbub, Light meson form factors at near physical masses, Phys. Rev. D 91, 074503 (2015).

[27] E. V. Lushevskaya, O. E. Solovjeva, and O. V. Teryaev, Determination of the properties of vector mesons in external magnetic field by quenched SU(3) lattice QCD, J. High Energy Phys. 09 (2017) 142.

[28] D. G. Gudiño and G. T. Sánchez, Determination of the magnetic dipole moment of the $\rho$ meson using four-pion electroproduction data, Int. J. Mod. Phys. A 30, 1550114 (2015).

[29] P. A. M. Dirac, Forms of relativistic dynamics, Rev. Mod. Phys. 21, 392 (1949).

[30] H. Leutwyler and J. Stern, Relativistic dynamics on null plane, Ann. Phys. (N.Y.) 112, 94 (1978).

[31] B. D. Keister and W. N. Polyzou, Relativistic Hamiltonian dynamics in nuclear and particle physics, Adv. Nucl. Phys. 20, 225 (1991).

[32] F. Coester, Null-Plane Dynamics of Particles and Fields, Prog. Part. Nucl. Phys. 29, 1 (1992).

[33] A. F. Krutov and V. E. Troitsky, Instant form of Poincaréinvariant quantum mechanics and description of the structure of composite systems, Phys. Part. Nucl. 40, 136 (2009).

[34] A. F. Krutov, R. G. Polezhaev, and V. E. Troitsky, Radius of the $\rho$ meson determined from its decay constant, Phys. Rev. D 93, 036007 (2016).

[35] A. F. Krutov and V.E. Troitsky, Relativistic instant-form approach to the structure of two-body composite system, Phys. Rev. C 65, 045501 (2002).

[36] A. F. Krutov and V.E. Troitsky, Relativistic instant-form approach to the structure of two-body composite system. Nonzero spin, Phys. Rev. C 68, 018501 (2003).

[37] A. F. Krutov and V. E. Troitsky, On a possible estimation of the constituent-quark parameters from Jefferson Lab experiments on the pion form factor, Eur. Phys. J. C 20, 71 (2001).

[38] J. Volmer et al. (Jefferson Lab $F_{\pi}$ Collaboration), Measurement of Charged Pion Electromagnetic Form-Factor, Phys. Rev. Lett. 86, 1713 (2001).

[39] T. Horn et al. (Jefferson Lab $F_{\pi}-2$ Collaboration), Determination of Charged Pion Form Factor at $Q^{2}=1.60$ and $Q^{2}=2.45(\mathrm{GeV} / c)^{2}$, Phys. Rev. Lett. 97, 192001 (2006).

[40] V. Tadevosyan et al. (Jefferson Lab $F_{\pi}$ Collaboration), Determination of pion charge form factor for $Q^{2}=$ 0.60-1.60-(GeV/c)2 $)^{2}$ Phys. Rev. C 75, 055205 (2007).

[41] H. P. Blok et al. (Jefferson Lab $F_{\pi}$ Collaboration), Charged pion form factor between $Q^{2}=0.60$ and $2.45 \mathrm{GeV}^{2}$. I. Measurmente of the cross section for the ${ }^{1} H\left(e, e^{\prime} \pi^{+}\right) n$ reaction, Phys. Rev. C 78, 045202 (2008).

[42] G. M. Huber et al. (Jefferson Lab $F_{\pi}$ Collfboration), Charged pion form factor between $Q^{2}=0.60$ and $2.45 \mathrm{GeV}^{2}$. II. Determination of, and results for, the pion form factor, Phys. Rev. C 78, 045203 (2008).

[43] A. F. Krutov, V. E. Troitsky, and N. A. Tsirova, Nonperturbative relativistic approach to pion form factors: Predictions for future JLab experiments, Phys. Rev. C 80, 055210 (2009).

[44] A. F. Krutov and V. E. Troitsky, Asymptotic estimates of the pion charge form factor, Theor. Math. Phys. 116, 907 (1998).
[45] S. V. Troitsky and V. E. Troitsky, Transition from a relativistic constituent-quark model to the quantum-chromodynamical asymptotics: a quantitative description of the pion electromagnetic form factor at intermediate values of the momentum transfer, Phys. Rev. D 88, 093005 (2013).

[46] S. V. Troitsky and V. E. Troitsky, Constraining scenarios of the soft/hard transition for pion electromagnetic form factor of $12 \mathrm{GeV}$ Jefferson Lab experiments and of the electronion collider, Phys. Rev. D 91, 033008 (2015).

[47] A. F. Krutov and V. E. Troitsky, Deuteron tensor polarization component $T_{20}\left(Q^{2}\right)$ as a crucial test for deuteron wave functions, Phys. Rev. C 75, 014001 (2007).

[48] A. F. Krutov and V. E. Troitsky, Extraction of the neutron charge form factor from the charge form factor of deutron, Eur. Phys. J. A 16, 285 (2003).

[49] A. F. Krutov, V. E. Troitsky, and N. A. Tsirova, Asymptotic behavior of the deuteron form factors in the two-nucleon model and electron scattering experiment at $\mathrm{GeV}$ energies at JLab, Phys. Rev. C 78, 044002 (2008).

[50] A. F. Krutov, S. V. Troitsky, and V. E. Troitsky, The $K$-meson form factor and charge radius: linking low-energy data to future Jefferson Laboratory measurments, Eur. Phys. J. C 77, 464 (2017).

[51] B. Bakamjian and L.H. Thomas, Relativistic particle dynamics. II, Phys. Rev. 92, 1300 (1953).

[52] A. A. Cheshkov and Yu. M. Shirokov, Invariant parametrization of local operators, Zh. Eksp. Teor. Fiz. 44, 1982 (1963) [Sov. Phys. JETP 17, 1333 (1963)].

[53] V. E. Troitsky and Yu. M. Shirokov, Relation between jumps at kinematic and anomalous cuts and the $S$-matrix at a mass shell, Theor. Math. Phys. 1, 164 (1969).

[54] V. P. Kozhevnikov, V. E. Troitsky, S. V. Trubnikov, and Yu. M. Shirokov, On the relationship between the deuteron form factors and the physical $S$-matrix. I, Theor. Math. Phys. 10, 30 (1972).

[55] A.F. Krutov and V.E. Troitsky, Construction of formfactors of composite systems by a generalized WignerEckart theorem for the Poincaré group, Theor. Math. Phys. 143, 704 (2005).

[56] A. F. Krutov, M. A. Nefedov, and V. E. Troitsky, Analytic continuation of the pion form factor from the spacelike to the timelike domain, Theor. Math. Phys. 174, 331 (2013).

[57] S. J. Brodsky and J. R. Hiller, Universal properties of the electromagnetic interactions of spin-one systems, Phys. Rev. D 46, 2141 (1992).

[58] R. G. Arnold, C. E. Carlson, and F. Gross, Elastic electrondeuteron scattering at high energy, Phys. Rev. C 21, 1426 (1980).

[59] S. J. Brodsky and F. Schlumpf, The impact of QCD and light-cone quantum mechanics on nuclear physics, Prog. Part. Nucl. Phys. 34, 69 (1995).

[60] F. Coester and W. N. Polyzou, Charge form factors of quarkmodel pions, Phys. Rev. C 71, 028202 (2005).

[61] F. Cardarelli, I. L. Grach, I. M. Narodetskii, E. Pace, G. Salmé, and S. Simula, Charge form factor of $\pi$ and $K$ mesons, Phys. Rev. D 53, 6682 (1996).

[62] K. A. Olive et al. (Particle Data Group Collaboration), Review of Particle Physics, Chin. Phys. C 38, 090001 (2014). 
[63] C. S. Mello, A. N. da Silva, J. P. B. C. de Melo, and T. Frederico, Light-front spin-1 model: Parameters dependence, Few-Body Syst. 56, 509 (2015).

[64] A. Ballon-Bayona, G. Krein, and C. Miller, Strong couplings and form factors of charmed mesons in holographic QCD, Phys. Rev. D 96, 014017 (2017).

[65] A. F. Krutov, Electroweak properties of light mesons in the relativistic model of constituent quarks, Phys. At. Nucl. 60, 1305 (1997).

[66] H. R. Grigoryan and A. V. Radyushkin, Structure of vector mesons in holographic model with linear confinement, Phys. Rev. D 76, 095007 (2007).

[67] J.P. B. C. de Melo, T. Frederico, and C.-R. Ji, Spin-1 particles and perturbative QCD, arXiv:1801.07631 v1.

[68] J.P. Lees et al. (BABAR Collaboration), Measurement of the $e^{+} e^{-} \rightarrow \pi^{+} \pi^{+} \pi^{-} \pi^{0} \pi^{0} \quad$ cross section using initial—state radiation at BABAR, Phys. Rev. D 96, 092009 (2017).

[69] O. V. Teryaev, Gravitational form factors and nucleon spin structure, Front. Phys. 11, 111207 (2016).

[70] T. T. Wu and C. N. Yang, Some speculations concerning high-energy large momentum transfer processes, Phys. Rev. B 137, B708 (1965).

[71] T. T. Chou and C. N. Yang, Model of elastic high-energy scattering, Phys. Rev. 170, 1591 (1968).

[72] B. Povh and J. Hüfner, Geometric Interpretation of HadronProton Total Cross Sections and Determination Hadronic Radii, Phys. Rev. Lett. 58, 1612 (1987).

[73] B. Povh and J. Hüfner, Systematics of strong interaction radii for hadrons, Phys. Lett. B 245, 653 (1990).

[74] M. Gourdin, Weak and electromagnetic form factors of hadrons, Phys. Rep. 11, 29 (1974). 